\title{
A Model and Guidelines for the Interface Design Process for Adaptive Web Applications (IDPAWA)
}

\author{
Claudia Regina Batista ${ }^{1}$, Vania Ribas Ulbricht ${ }^{1}$, and Adhemar Maria do Valle Filho ${ }^{2}$ \\ ${ }^{1}$ Federal University of Santa Catarina, Department of Graphic Expression, Trindade, \\ 88040-900 Florianópolis - Santa Catarina, Brazil \\ claudia.batista@ufsc.br, vulbricht@gmail.com \\ ${ }^{2}$ University of Vale do Itajaí, Computer Science, Itajaí - Santa Catarina, Brazil \\ adhe.valle@gmail.com
}

\begin{abstract}
This paper shows a model and guidelines for the Interface Design Process for Adaptive Web Applications (IDPAWA) proposed to guide and aid the designer on taking decisions during the interfaces development. The model schematically describes five steps of an interactive process: analysis, concept, development, prototype and test. In order to support the model, eight guidelines were developed to deal with the requirements and configuration of adaptive techniques.
\end{abstract}

Keywords: Design Process, User Interface, Visual Design, Adaptive Web Applications.

\section{Introduction}

The World Wide Web, or simply Web, became a powerful electronic vehicle of global proportions. The interaction, the non-linearity, the freedom of navigating thru a wide informational space and the media plurality made Web attractive and engaging. However, there is still a long way to be pursued regarding usability.

Problems related to users disorientation and cognitive overload resulted on the growth of interest on researches that present solutions for a better working when it comes to users' variety. One alternative that promotes a better assistance to the heterogeneity of profiles and users is the Adaptive Hypermedia. According to Brusilovsky (2007): “Adaptive hypermedia systems (AHS) offer an alternative to the traditional 'one-size-fits-all' hypermedia and Web systems by adapting to the goals, interests, and knowledge of individual users as they are represented in the individual user models" [3]. These environments potentiate an approach focused on the user, since the system adapts the visible aspects according to the 'user model' (build based on the user's information) creating an interface that offers appropriated information, with the right layout for each user [10].

On the other hand, it is noticeable that the design and development of Web applications and services that meet the needs and requirements of as many diverse users as possible is still a difficult and demanding task [10]. 


\section{A Contribution to Interface Design Process for Adaptive Web Applications (IDPAWA)}

\subsection{Research Problem}

The design and implementation of adaptive hypermedia systems or Adaptive Web Applications represents a time-consuming and difficult job, which make them complex and expensive systems [10].

There are support tools to interface design such as ISO standards [7] [8] [9] and others described on the literature [1] [5] [11] [13] [14] [15] that establishes rules, principles, guidelines, criteria, recommendations, methods and are useful to assist the designers by contemplating from the conception to the evaluation of interfaces along with the user. However, these support tools to interface design were not conceived under the perspective of Adaptive Hypermedia, therefore they don't present guidance on the specificities of these systems (as example, the requirements for application of content, navigation and presentation adaptation techniques) [2].

According to the presented context, it is observed the lack of support to Interface Design Process for Adaptive Web Applications. The incipient orientations to interface design induce the designers to adjust the generic orientations to their needs and adopt inappropriate developing processes, which might result in interfaces without usability, with communication failures, compromised interaction, inappropriate layout and others. "If" poorly projected, the interface impairs the functionality and endangers the quality of web adaptive applications [2].

The lack and/or incipience of orientations to the interface design to Adaptive Web Applications constitute a lacuna of knowledge in this area. Therefore, it is proposed a research which objectives are presented on the following lines.

\subsection{Objective and Methods}

The main objective of research was the development of a model and guidelines to Interface Design Process for Adaptive Web Applications (IDPAWA) in order to guide and support the web interface designer on taking decisions during design process.

This applied research approached the problem in a qualitative way and adopted the technic procedures of bibliographic research and study of case. The research was accomplished in four phases: survey, analysis, proposition and validation.

- In the survey phase, it was performed a bibliographic research and with the collected data it was possible to consolidate the theoretical foundation and establish the "state of art" in Adaptive Web Applications.

- In the analysis phase, the collected data on the bibliographic research was analysed and by that way it was possible to define the interfaces' properties to Adaptive Web Applications, as well as identify the designer's specific tasks during the development of an adaptive website.

- In the proposition phase, the qualitative research was accomplished by crossing the information that was collected on the bibliographic research and by an inductive analysis of data related to the properties and requirements of Adaptive Web 
Applications where it was possible to outline a task flow to be accomplished by the designer during the IDPAWA. Initially it was proposed a model that schematically describes a structure of concepts and steps, in order to guide the designer during the IDPAWA. The guidelines were established as a set of instructions with the objective of supporting the proposed model and guide the designers thru the configuration of adaptive techniques and interface requirements.

- In the validation phase, the Model and Guidelines to IDPAWA were available to designers that composed the sample to be validated during the interface development to the adaptive website. The population research was composed of web interface designers. The sample was determined by the inclusion and exclusion criteria. As inclusion criteria, were defined: act professionally as a web interface designer; and agree with being a volunteer on the project. Analogously, the exclusion criteria were: having experience for less than two years on developing web interfaces.

\subsection{Results}

The interface characteristics, the task flow of the interface designer, the proposal for a model of design process and guidelines constitute the research results and are presented below.

\section{The Identification of Interface Characteristics for Adaptive Web Applications.}

The essential characteristics that define an interface to Adaptive Web Applications are presented on the concept board ${ }^{1}$ (figure 1).

The elements presented on the concept board are described next:

- TRANSFORMABLE: the Interface for Adaptive Web Applications transforms itself. In other words, it changes itself in order to fit preferences, needs and users' characteristics. The adaption methods and techniques allow the necessary transformations.

- ASSEMBLE and DISASSEMBLE: the Interface for Adaptive Web Applications must be a dynamic interface. It is possible to make an analogy with the LEGO ${ }^{\mathrm{TM}}$ toy: "with the same pieces" it is possible to assemble different solutions; and with the same content (stored in the database) it is possible to assemble dynamically different ways for interaction and presentation of information. The fact that the content is separated from presentation/formatting is also significant, which provides better accessibility.

- FLEXIBLE: the Interface for Adaptive Web Applications must reflect the system's flexibility, providing ways of action for the user in order to take command (he can skip the system's recommendations and redefine his own way of information visualization if he so desires). There also must be flexibility to resize it in different screens and resolutions.

\footnotetext{
${ }^{1}$ On a concept board it is visually and/or verbally presented an idea for a product or service; usually described its' attributes or benefits.
} 
- SPEEDY: the Interface for Adaptive Web Applications must load instantly. The interfaces with valid code, accessible code, semantically correct code (Web Standards) are going be rapidly interpreted by browsers.

- LIGHT: the Interface for Adaptive Web Applications must be composed by light image, videos and audio files, in order to provide speed when loading it.

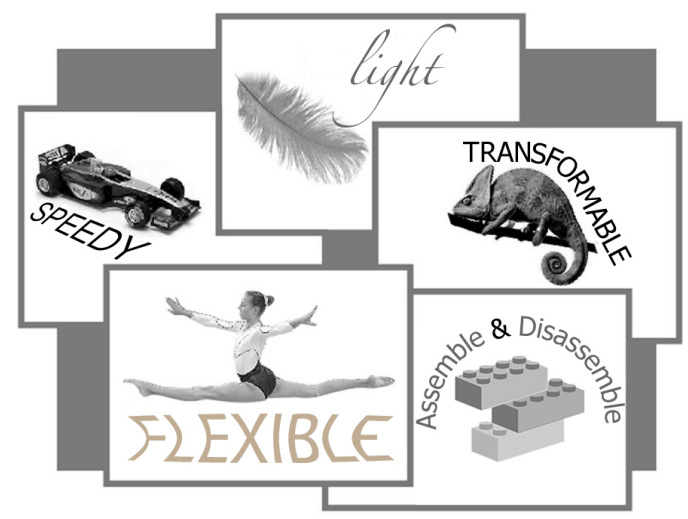

Fig. 1. Interface Concept Board for Web Applications (Source: Batista, 2008, p. 64)

Interface Designer's Task Flow to Adaptive Web Applications. During the development of an Adaptive Web Application, the interface designer works along with the multidisciplinary developing team. The interface designer must analyse the software artefacts (domain, navigation and adaption models) produced by other team members, in order to extract the requirements to the interface project.

From the investigation and data analysis concerning the developing process of Adaptive Web Hypermedia Systems and Adaptive Applications, from the developed activities by the multidisciplinary team and the characteristics of Interface for Web Applications, it was possible to outline a task flow to be accomplished by the designer during the IDPAWA.

The task flow presented on Figure 2 was the fist step to develop the Model for IDPAWA, since it was necessary to understand which activities are involved with the interface design for Adaptive Web Applications, in order to visualize the complete developing process. 


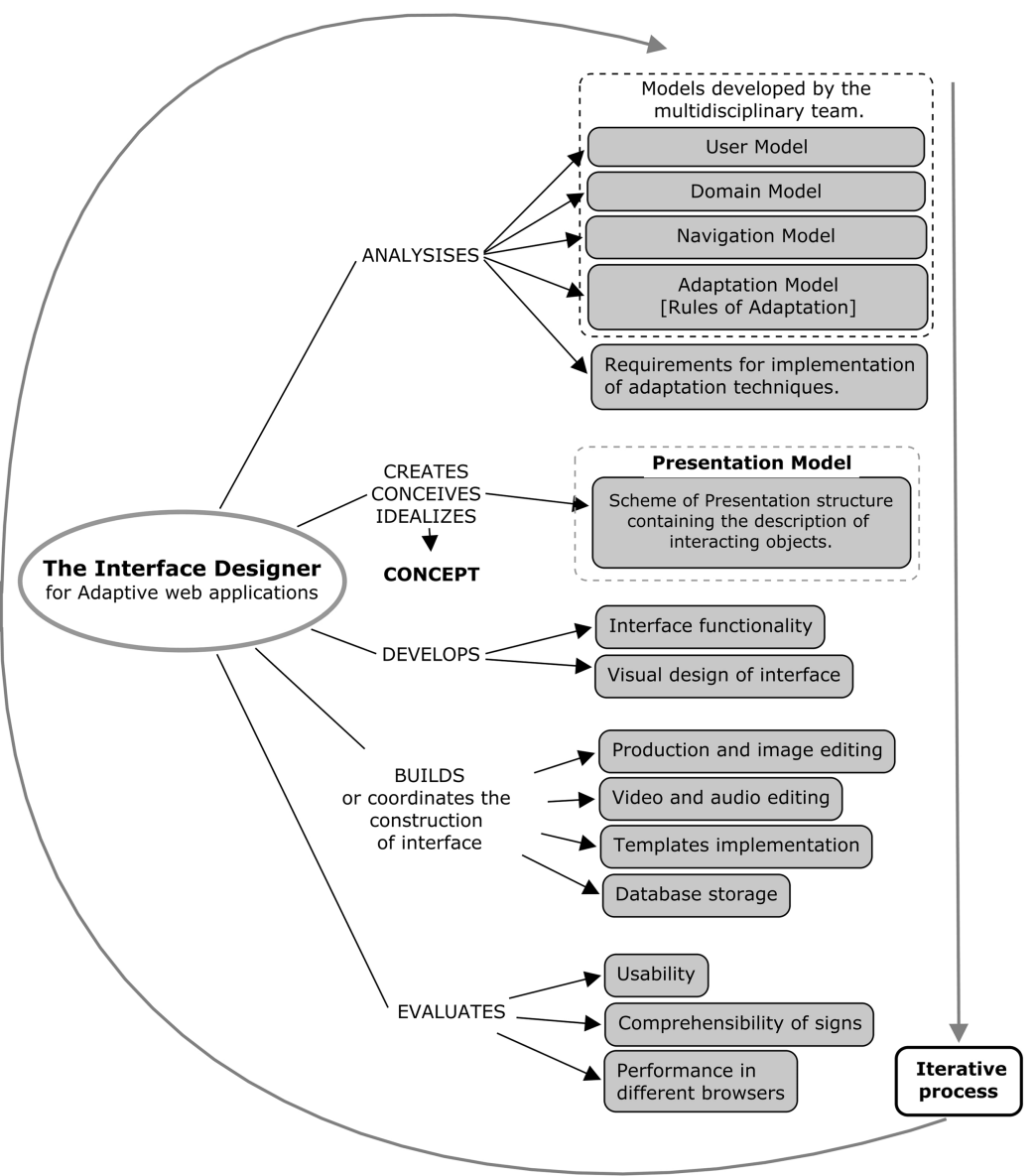

Fig. 2. Interface designer's task flow during the IDPAWA (Source: Batista, 2008, p. 66)

Process Model of Interface Design for Adaptive Web Applications. Considering that the interface developing process is not a top-down activity, a model that describes an iterative sequence of steps was proposed, in order to guide the designer during the process of Interface Design for Adaptive Web Applications (IDPAWA). The model presented on Figure 3 allows the designer to obtain a global vision of the design process and accomplished a structured, systematic and organized job. The analysis initiates the sequence; when coursing the clockwise direction, the transition for the following steps is made: concept, development, prototype and test. 


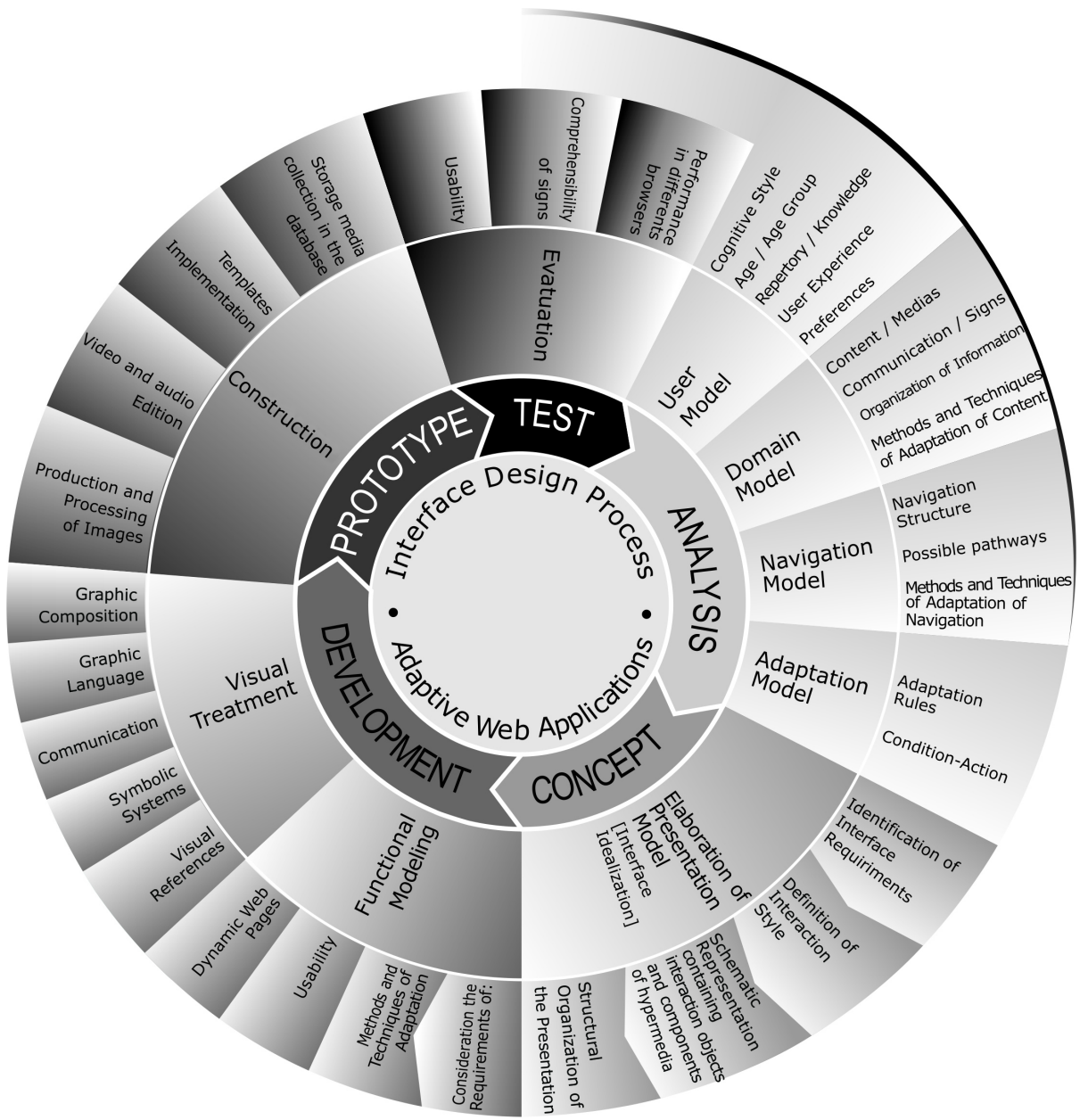

Fig. 3. Model of Interface Design Process for Adaptive Web Applications (Source: Batista, 2008, p. 67)

Next it is made a description of steps from the Interface Design Process for Adaptive Web Applications:

- ANALYSIS step: While on the development of non-adaptive systems the designer starts the process by the data collection step, on IDPAWA he will begin his activity analysing the models made by the multidisciplinary team involved on the Adaptive Web Application project. It means that the multidisciplinary team has already accomplished the data collection step to build the user's models, of domain, navigation and adaption. They have also already defined the methods and adaption techniques that will be utilized. 
Then, the designer's task is to understand how the system will operate, the context of use and the adaption techniques requirements, in order to establish goals and objectives of interface to the Adaptive Web Application.

- CONCEPT step: After understanding how the Adaptive Web Application will be, the designer is going to list the interface requirements, define the interaction style; in other words, will idealize the interface. After it is done, the designer will schematically represent it by describing the location of interaction objects, hypermedia elements and the structural organization of presentation. This schematic representation is the Presentation Model.

- DEVELOPMENT step: After building the Presentation Model, the design will develop the interface. He will make the functional modeling, where is necessary to generate interface elements in order to attend the requirements of methods and adaption techniques, of usability and dynamic web pages. To accomplish the visual treatment (create interface layout) the designer must search visual references in agreement with the user model, generate symbolic and communicational systems, identify the graphic language pertinent to the user model and define the graphic composition of the interface.

- PROTOTYPE step: If the designer has skills and ability to implement the prototype, then within the multidisciplinary team he can assume the programmer function. Otherwise he can coordinate the prototype construction. In this step, it is accomplished the production and treatment of images, video and audio edition, templates implementation and storage media collection in the database.

- TEST step: The same interface evaluation processes of non-adaptive systems can be applied on Adaptive Web Applications. In this step it should be accomplished usability tests (before and after the implementation), of comprehensibility of signs (icons and others) and the application performance in different browsers.

After accomplishing a cycle, which means accomplishing the test step, the process is not yet finished, because based on the evaluation feedback the cycle can be repeated as many times as necessary.

The proposed model reflects the logic of the Interface Design Process for Adaptive Web Applications, but does not guarantee the project's success. It is relevant to mention that the final quality of the interface is also related to some of the designer attributes, such as knowledge, abilities, experience and sensibility to create visual communication and accomplish the visual treatment.

Guidelines for Interface Design for Adaptive Web Applications. According to the IDPAWA model, the designer must identify the methods and adaption techniques that will be used on adaptive systems and establish ways to enable them on the interface.

The designer will work directly with adaption techniques, since they implement the adaption methods [4] [2]. Therefore, the designer needs to know the characteristics 
and particularities of adaption techniques. To help the designer task and support the proposed model, guidelines for IDPAWA were developed. The Guidelines ${ }^{2}$ for Interface Design for Adaptive Web Applications are a set of interactions that deal with requirements and the adaption techniques configuration. It was made one guideline for each one of the adaption techniques related below:

- Strechtext - Content Adaption Technique

- Conditional Fragment - Content Adaption Technique

- Page Variants - Content Adaption Technique

- Direct Orientation - Navigation Adaption Technique

- Links Note - Navigation Adaption Technique

- Hiding/Removal of Links - Navigation Adaption Technique

- Page Variants - Presentation Adaption Technique

The guidelines present descriptions of adaption techniques concerning function, objective, properties, operation description with examples, strategy and requirements for the interface design. However, some guidelines are longer than others, since some adaption techniques require specific interface elements. It brings more orientations and examples to support the interface designer. The adaption techniques that require more interface elements development are the Stretchtext (Content Adaption Techniques), the Direct Orientation and the Links Note (both are Navigation Adaption Techniques). They demand the development of specific areas on the interface, of interaction objects and visual treatment in order to call the user's attention. However, the content adaption techniques Conditional Fragment and Page Variants; and the navigation adaption techniques Hiding/Removal of Links and Links Ordination do not require the interface elements development, which means that they do not infer on the interface configuration.

Validation. Seven designers (whose profile were compatible with the sample criteria) participated in the research to validate the model and guidelines. The Model and the Guidelines for IDPAWA were provided to the seven designers during the interface design for an adaptive website entitled "Diferente todo mundo é!" / "Everyone is different!" ${ }^{3}$.

According to designers, the model shows clearly the design process. It was easy to understand all steps. In respect guidelines, it was perceived that the interface designers needed more data. At the initial stage of the work, it was difficult for designers to understand the characteristics of adaptation techniques, because the guidelines had only a description about them. They have suggested the inclusion of examples with graphic elements that should appear on the interface.

2 The Guidelines were commented briefly, in order to fit the size of this article. In Batista (2008) the guidelines are fully presented.

3 "Everyone is different!" is an Adaptive Web Application that offers information about Down Syndrome. The content, navigation and presentation are adapted according to the "user model". 
The designers' feedback led to revision, restructuring and improvement of the guidelines. It is important to emphasize that this phase of research was crucial to make guidelines more complete. After the application, it was noticeable that the model and guidelines for IDPAWA accomplished its' purpose (provide the necessary support during the project), since the designers who were unaware of this particular area were able to develop the interface for Adaptive Web Application.

\section{The Personalised Interfaces Created for the Website about Down Syndrome.} Besides the Model and Guidelines for IDPAWA, the designers received information about the project "Everyone is different!" and software artefacts produced by the development team, such as: user model, domain model, navigation model and adaptation rules. They analysed these documents to understand the logic of the system. This website provides the adaptation of content, navigation and presentation to three user profiles: child, teenager and adult. In this project was used the adaptation techniques presented next:

- The Presentation Adaption Technique - Page Variants: it provides a mode of presentation and interaction consistent with the cognitive characteristics of each user class. Thus, to meet the attributes of each user class, the designers created three distinct modes of presentation, i.e., the child template, the teenager template and adult template, presented below.

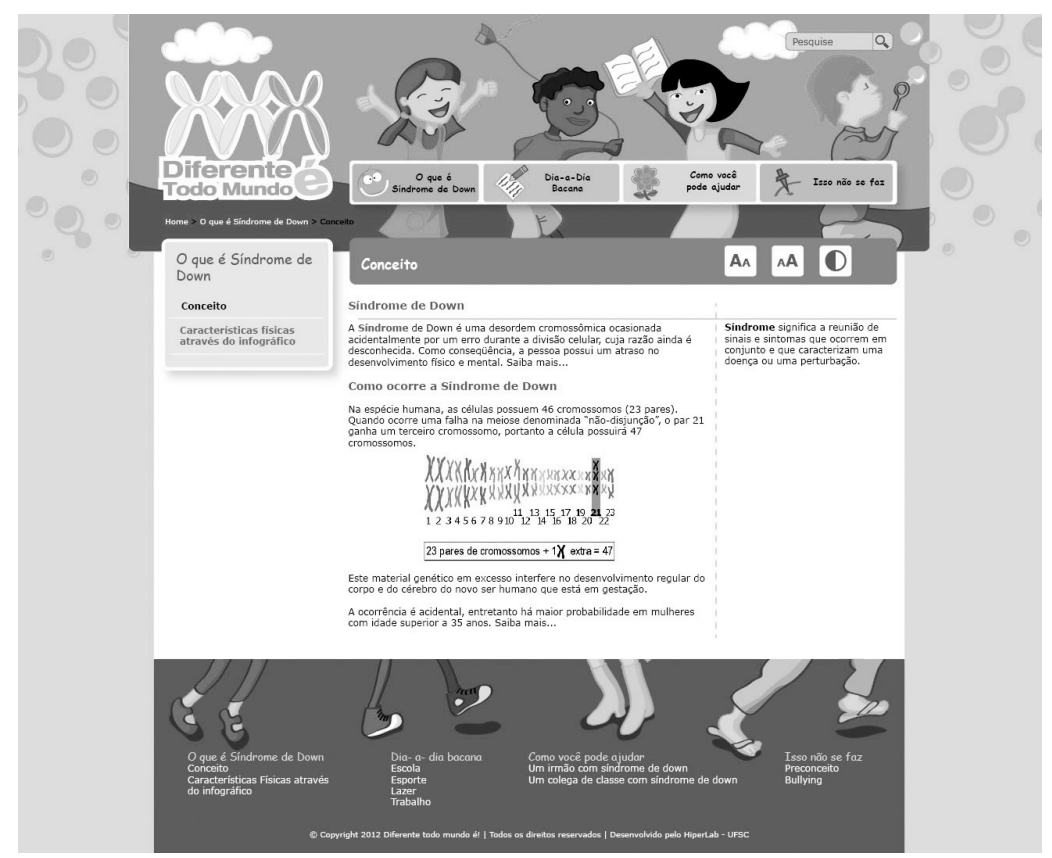

Fig. 4. Content, Navigation and Presentation personalized for child (Source: Reginaldo, 2012) 


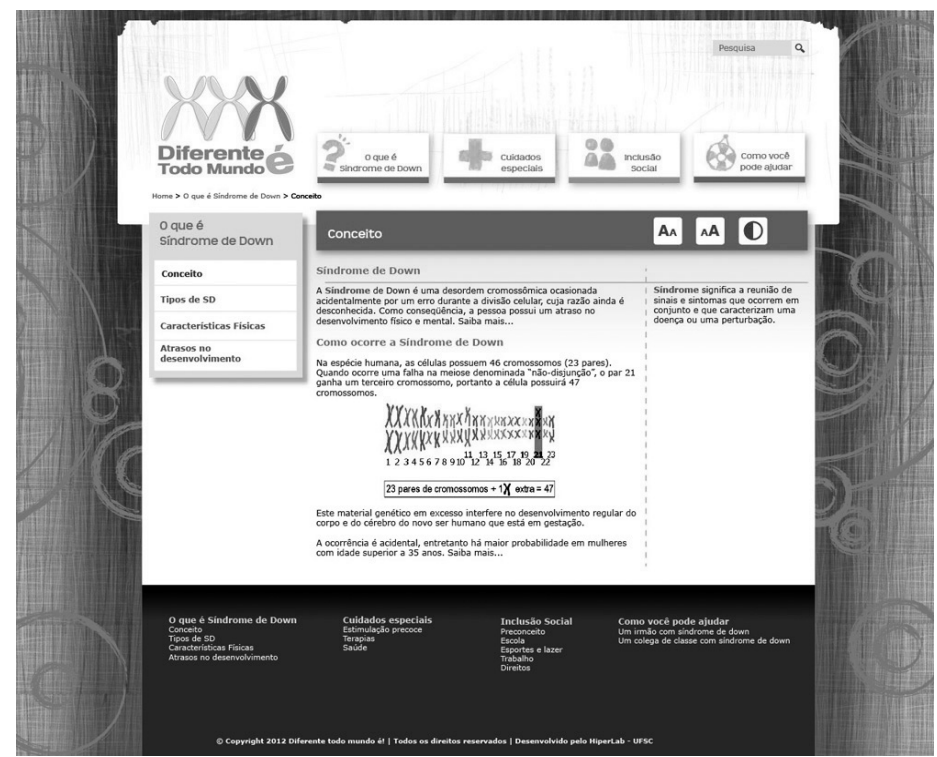

Fig. 5. Content, Navigation and Presentation personalized for adolescent (Source: Reginaldo, 2012)

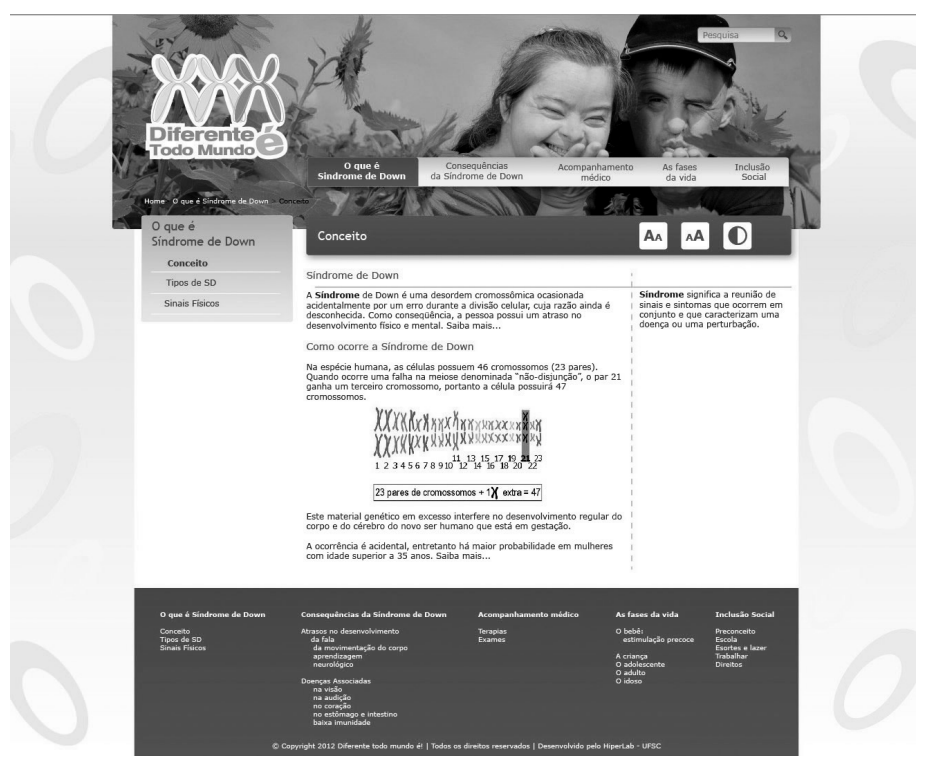

Fig. 6. Content, Navigation and Presentation personalized for adult (Source: Reginaldo, 2012)

- The Content Adaption Technique - Strechtext: it includes additional / complementary explanations, definitions, concepts, examples, by expandable paragraphs and/or marginal notes. The Figure 7 shows an example of use of Strechtext. 


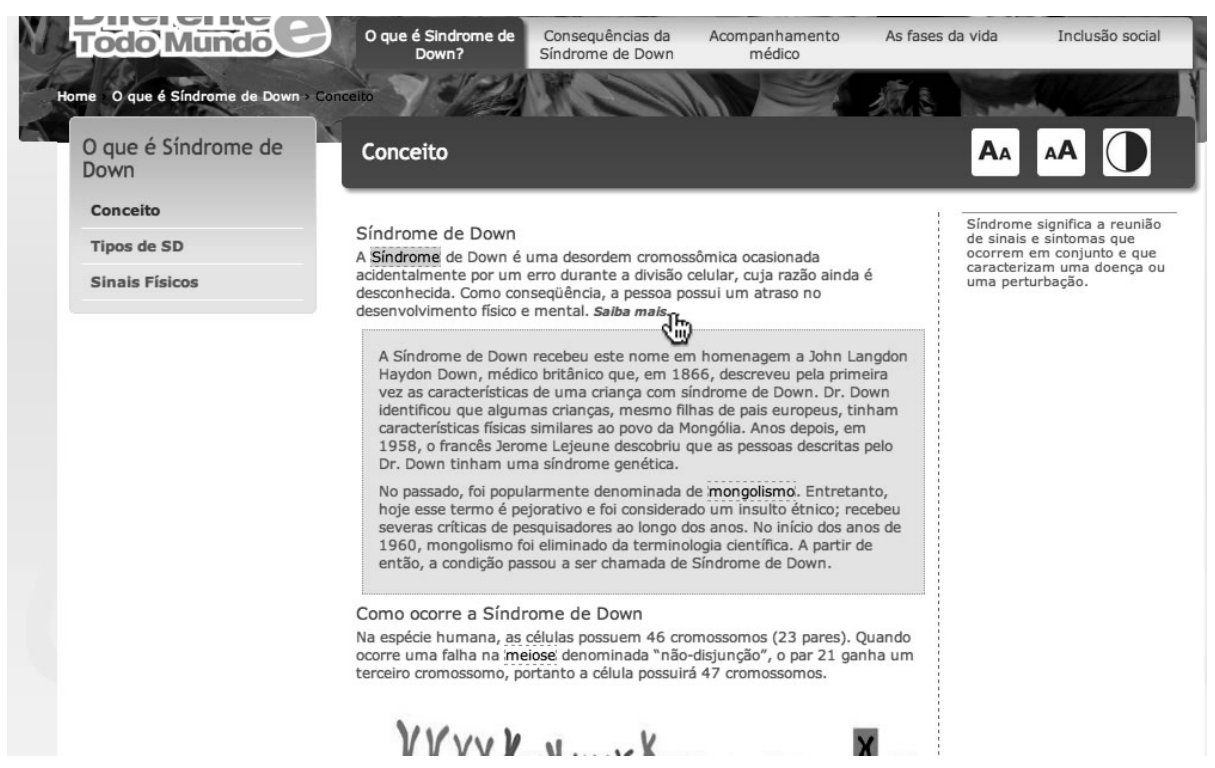

Fig. 7. Complementary information is added in expandable paragraphs (Source: Reginaldo, 2012)

\section{Final Considerations}

The scope of this study consisted on the proposition of a solution to guide de designer during the interface design process for Adaptive Web Applications, contemplating aspects regarding the dynamic structure of web document, graphic presentation quality, implementation technology, the interface elements performance that provide navigation and adaptive presentation.

The model and guidelines for IDPAWA bring contributions in order to facilitate the tasks of professionals from the Design and Communication and Information Technology areas.

However, it was noticeable that the authors of content/specialists on domain (professionals who were not graduated on computation) also need orientations concerning the use of content adaption techniques. Therefore, the possibility of developing guidelines dedicated to authors of content is foreseen.

It is also necessary to investigate if the utilized solutions by the adaptive techniques provide accessibility to the web document.

There is space for new researches in the usability sphere of adaptive websites. The adaptive hypermedia development represents a promising area of investigations. However, in Brazil, the researches in this area are still in an early stage. Therefore, it is the interested people's mission to participate of this new model of information, acquire knowledge, discuss and propose solutions that might contribute to the consolidation of this new media. 


\section{References}

1. Bastien, J.M.C., Scapin, D.L.: Human factors criteria, principles, and recommendations for HCI: methodological and standardization issues, Internal Report, Institut national de recherche en informatique et en automatique - INRIA, Rocquencourt, France (1993)

2. Batista, C.R.: Model and guidelines for the design process of adaptive web interface. Florianópolis. Thesis (Doctorate in Engineering and Knowledge Management). Federal University of Santa Catarina (2008)

3. Brusilovsky, P.: Adaptive Navigation Support. In: Brusilovsky, P., Kobsa, A., Nejdl, W. (eds.) Adaptive Web 2007. LNCS, vol. 4321, pp. 263-290. Springer, Heidelberg (2007)

4. Doulgeraki, C., Partarakis, N., Mourouzis, A., Stephanidis, C.: Adaptable Web-based user interfaces: methodology and practice. eMinds: International Journal on Human-Computer Interaction I(5), 79-110 (2009)

5. Garrett, J.J.: The elements of user experience: user centered design for the web, 2nd edn. New Riders, New York (2010)

6. Gena, C., Weibelzahl, S.: Usability Engineering for the Adaptive Web. In: Brusilovsky, P., Kobsa, A., Nejdl, W. (eds.) Adaptive Web 2007. LNCS, vol. 4321, pp. 720-762. Springer, Heidelberg (2007)

7. ISO 13407. Human-centred design processes for interactive systems (1999) Revised by: ISO 9241-210 (2010)

8. ISO 9241-11. Ergonomic requirements for office work with visual display terminals (VDTs) - Part 11: Guidance on usability (1998)

9. ISO 9241-12. Ergonomic requirements for office work with visual display terminals (VDTs) - Part 12: Presentation of information (1998)

10. Koch, N.P.: Software Engineering for Adaptive Hypermedia Systems: Reference Model, Modeling Techniques and Development Process. Munich, Thesis (Doctor of the Natural Sciences at the Faculty for Mathematics and Computer Science), Ludwig Maximilians University Munich (2000), http://www.pst.informatik.uni-muenchen. de/ kochn/PhDThesisNoraKoch.pdf

11. Nielsen, J., Loranger, H.: Prioritizing Web Usability. New Riders Press, Berkeley (2006)

12. Reginaldo, T.: Personalised interfaces design for adaptive website "Everyone is different!'. Florianópolis. Monograph (Bachelor's degree in Design) Federal University of Santa Catarina (2012)

13. Rosenfeld, L., Morville, P.: Information Architecture for the World Wide Web, 2nd edn. O’Reilly, Sebastopol (2002)

14. Sharp, H., Rogers, Y., Preece, J.: Interaction Design: Beyond Human-Computer Interaction, 3rd edn. John Wiley \& Sons, Inc., Chichester (2011)

15. Shneiderman, B., Plaisant, C.: Designing the user interface: Strategies for effective Human-Computer Interaction, 5th edn. Addison Wesley Longman, Inc., Berkeley (2009) 